\title{
Atomic Bell measurement via two-photon interactions
}

\author{
Carlos A. González-Gutiérrez ${ }^{1}$ and Juan Mauricio Torres ${ }^{2}$ \\ ${ }^{1}$ Instituto de Ciencias Físicas, Universidad Nacional Autónoma de México, \\ Apartado Postal 48-3, Cuernavaca, Morelos 62251, México \\ ${ }^{2}$ Instituto de Física, Benemérita Universidad Autónoma de Puebla, Apartado Postal J-48, Puebla 72570, México
}

(Dated: February 27, 2019)

\begin{abstract}
We introduce a complete Bell measurement on atomic qubits based on two photon interactions with optical cavities and discrimination of coherent states of light. The dynamical system is described by the Dicke model for two three-level atoms interacting in two-photon resonance with a single-mode of the radiation field, which is known to effectively generate a non-linear two-photon interaction between the field and two states of each atom. For initial coherent states with large mean photon number, the field state is well represented by two coherent states at half revival time. For certain product states of the atoms, we prove the coherent generation of GHZ states with two atomic qubits and two orthogonal Schrödinger cat states as a third qubit. For arbitrary atomic states, we show that discriminating the two states of the field corresponds to different operations in the Bell basis of the atoms. By repeating this process with a second cavity prepared in a phase-shifted coherent state, we demonstrate the implementation of a complete Bell measurement. Experimental feasibility of our protocols is discussed for cavity-QED, circuit-QED and trapped ions setups.
\end{abstract}

\section{INTRODUCTION}

Bell measurements are crucial to implement quantum information protocols such as quantum teleportation, superdense coding, and entanglement swapping [1,2]. These protocols play a key role in the nodes of a quantum repeater and to establish long-distance communication in a quantum network $[3,4]$. In a complete Bell measurement a two-qubit system is probabilistically projected onto one of the four Bell states. For photonic qubits, it is possible to identify two of the four Bell states, i.e. a 50\%-efficient Bell measurement, using interference effects with linear optics [5-7]. The capability to surpass this limit relies either on more resourceful techniques $[8,9]$ or on higher order optical interactions [10] that report low efficiency in the experiment [11]. In the case of atomic qubits, most experimental realizations of quantum teleportation consider the implementation of a complete Bell measurement through entangling gates, such as a controlled-NOT or controlled-phase gate, that together with single qubit gates can map Bell sates onto product states in the computational basis [12-14]. A problem with this approach, however, is that high fidelity two-qubit gates are still experimentally difficult to achieve [15-17].

Motivated by the hybrid quantum repeater [3] that employs material qubits and multiphoton coherent signals, a recently proposed alternative is to explore atom-photon interaction models that directly generate Bell states of the atoms correlated with states of the field. Using this approach, it was shown that unambiguous Bell state projections can be implemented within the framework of the two-atom Tavis-Cummings model [18-20]. There, the state of the atoms is postselected by projecting the states of the field onto nearly orthogonal coherent states. The great benefit is that the atomic states are not directly measured and their projection occurs as postselection of the measured field. An imperfect efficiency relies on the fact that initial coherent states of the field in the Tavis-Cummings model do not evolve coherently during the interaction [18, 21]. This is clearly manifested in the non-perfect revivals of Rabi oscillations of atomic observables, similar to the well known collapse and revival phenomena in the Jaynes-Cummings model [22]. The natural question that arises is whether an atom-field model presenting perfect revivals of Rabi oscillations could better assist in the postselection of atomic Bell states. The answer to this question turns out to be positive as we shall demonstrate.

In this paper we propose a complete atomic Bell measurement based on the two-photon two-atom Dicke model in the rotating wave approximation [23] that presents nearly perfect revivals of Rabi oscillations. Similar to previous work [18, 19], the states of the qubits are encoded in a pair of two-level atoms that interact resonantly and sequentially with the field inside two optical cavities and the atomic state is postselected by measuring the optical field. The considered two-photon atom-field interaction model was first introduced as a generalization of the Jaynes-Cummings model [24-26], and later extended for multiatomic systems [23, 27]. It has been proposed theoretically, but its experimental feasibility has been analyzed in well controllable quantum optical systems [28]. Although we focus on a cavity QED implementation, two-photon or two-phonon interactions have also been studied and proposed in circuit QED and trapped ions [29, 30], thus making our proposal attractive to other architectures involving matter-field interaction.

The paper is organized as follows. In Sec. II, we review the effective two-photon Dicke model as a limiting case of a general Dicke Hamiltonian of two three-level atoms and discuss the regime of validity in terms of the parameters of the system. In Sec. III, an approximate exact solution of the effective model in terms of coherent states of the field is presented and its validity is verified by comparing the fidelity with respect to exact numerical calculations. Collapse and revival of Rabi oscillations are studied in Sec. IV. The generation of tripartite entangled states is discussed in Sec. V. In Sec. VI the quantum protocol for a Bell-measurement is described in detail and numerically verified. We discuss possible experimental implementations in Sec. VII and our conclusions are given in Sec. VIII. 


\section{THE TWO-PHOTON MODEL}

In this section we briefly review the Dicke model in the rotating wave approximation at two-photon resonance with two identical three-level atoms (A and $\mathrm{B}$ ) that interact with one mode of the quantized electromagnetic field inside an optical cavity. The field couples an intermediate level $|\mathrm{i}\rangle$ with the ground state $|g\rangle$ and the excited state $|\mathrm{e}\rangle$ as depicted in Fig. 1. The frequency difference between ground and excited state is assumed to be tuned at twice the frequency of the cavity mode. Choosing units in which $\hbar=1$, the Hamiltonian describing the dynamics of the system can be written as

$$
H=\omega a^{\dagger} a+2 \omega S_{\mathrm{ee}}+(\omega+\Delta) S_{\mathrm{ii}}+V .
$$

The first term in the Hamiltonian describes the energy of the optical field and is written in terms of the bosonic annihilation and creation operators $a$ and $a^{\dagger}$. The second and third term represent the atomic energy of the excited and intermediate states, respectively. They are expressed trough the atomic collective operators

$$
S_{\mu v}=|\mu\rangle\left\langle\left. v\right|_{\mathrm{A}}+\mid \mu\right\rangle\left\langle\left. v\right|_{\mathrm{B}}, \quad \mu, v \in\{\mathrm{g}, \mathrm{i}, \mathrm{e}\} .\right.
$$

The last term in Eq. (1), $V$, describes the atom-field interaction which is assumed to fulfill the rotating-wave approximation (RWA) and therefore can be written as

$$
V=g_{\mathrm{g}} a S_{\mathrm{ig}}+g_{\mathrm{e}} a S_{\mathrm{ei}}+\text { H.c. }
$$

where $g_{\mathrm{e}}$ and $g_{\mathrm{e}}$ are the corresponding atom-field coupling strengths.

The detuning $\Delta$ between the frequency of the intermediate state and the frequency of the mode is assumed to be large compared with both coupling strengths that we consider of the same order of magnitude, namely $\Delta \gg g_{\mathrm{g}} \sim g_{\mathrm{e}}$. In this particular situation, it can be shown that the intermediate level can be approximately decoupled from the dynamics. To show this, we follow the method introduced in [23] and perform a small rotation of the Hamiltonian with the transformation

$$
e^{i G} H e^{-i G}, \quad G=\frac{g_{\mathrm{g}}}{\Delta} a S_{\mathrm{ig}}-\frac{g_{\mathrm{e}}}{\Delta} a S_{\mathrm{ei}}-\text { H.c. }
$$

Using the Baker-Campbell-Hausdorff $(\mathrm{BCH})$ expansion and neglecting terms of the order $g_{\mathrm{e}}\left(g_{\mathrm{e}} \sqrt{\left\langle a^{\dagger} a\right\rangle} / \Delta\right)^{2}$, one can obtain the following effective Hamiltonian

$$
H \approx \omega I+S+W
$$

which includes a two-photon interaction term

$$
W=g\left(a^{2} S_{\mathrm{eg}}+a^{\dagger 2} S_{\mathrm{ge}}\right), \quad g=-g_{\mathrm{e}} g_{\mathrm{e}} / \Delta
$$

The expansion also produces a Stark-shift contribution of the form

$$
S=-2 \frac{g_{\mathrm{g}}^{2}}{\Delta} I-\frac{g_{\mathrm{e}}^{2}-g_{\mathrm{g}}^{2}}{\Delta} a a^{\dagger} S_{\mathrm{ee}}+3 \frac{g_{\mathrm{g}}^{2}}{\Delta} S_{\mathrm{ee}}
$$

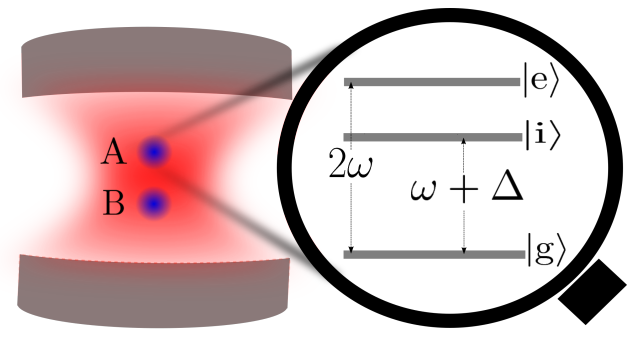

FIG. 1. Pictorial illustration of two three-level atoms (A and B) interacting at two-photon resonance with one mode of the radiation field inside an optical cavity. For large enough detuning $\Delta$ between the lower transition frequency and the frequency of the field, the intermediate state is decoupled from the dynamics leading to an effective two-photon interaction involving only states $|\mathrm{g}\rangle$ and $|\mathrm{e}\rangle$ of the atoms.

One can verify that the first term in Eq. (7) is a constant of motion that is given by

$$
I=a^{\dagger} a+2 S_{\mathrm{ee}}
$$

In principle, $I$ should also contain the term $S_{\mathrm{ii}}$, however one can safely omit it if the intermediate state is not initially populated. The effective Hamiltonian in (5) can be verified with the commutation relations $\left[G, S_{\mathrm{ii}}\right]=-V$ and $[G, V]=2 W+2 S$ that follow from $\left[S_{\mu \nu}, S_{\mu^{\prime} v^{\prime}}\right]=\delta_{\nu \mu^{\prime}} S_{\mu v^{\prime}}-\delta_{v^{\prime} \mu} S_{\mu^{\prime} v}$ using Eq. (2). Taking into account the order of the neglected terms, one can accurately describe the dynamics of the system using the effective Hamiltonian subjected to the following restriction in time

$$
g_{\mathrm{e}} t \ll \Delta^{2} / g_{\mathrm{e}}^{2}\left\langle a^{\dagger} a\right\rangle
$$

In order to further simplify the interaction, one can find conditions for which the photon-dependent Stark-shift term, second in Eq. (7), does not contribute to the dynamics. This part can be neglected if it is smaller than the omitted expressions in the truncated $\mathrm{BCH}$ expansion leading to the effective Hamiltonian in Eq. (5), which reduces to the condition $\left|g_{\mathrm{e}}^{2}-g_{\mathrm{g}}^{2}\right|<g_{\mathrm{e}}^{3} / \Delta$ quantifying the closeness between $g_{\mathrm{g}}$ and $g_{\mathrm{e}}$. With this in mind, the photon-independent Stark-shift, third in (7), is of the order of $g$, which can be neglected for large photon numbers compared with the order of $W$ given by $g\left\langle a^{\dagger} a\right\rangle$. Under these assumptions, one can reduce the Hamiltonian in Eq. (1) simply to

$$
H \approx(\omega+2 g) I+W
$$

As this Hamiltonian effectively describes the dynamics of the two atoms restricted to levels $|\mathrm{g}\rangle$ and $|\mathrm{e}\rangle$, in what follows we will solve the Schödinger equation for this Hamiltonian in the interaction picture with respect to the constant of motion $(\omega+2 g) I$ exploiting the fact that it commutes with the twophoton interaction, i.e., $[I, W]=0$. We stress that under the aforementioned assumptions the dynamics of the system is well described by the two-photon interaction term $W$ in Eq. (6). 


\section{SOLUTION OF THE DYNAMICAL EQUATION FOR LARGE PHOTON NUMBER}

In this section we derive an approximate analytical solution for the time-dependent state vector in the limit of large mean photon numbers. To this end, we consider initial states of the form $|\Psi\rangle=|\psi\rangle|\alpha\rangle$, where $|\psi\rangle$ is an arbitrary state of two two-level atoms, and where we have considered the photonic coherent state

$$
|\alpha\rangle=\sum_{n=0}^{\infty} p_{n}|n\rangle, \quad p_{n}=e^{-\frac{|\alpha|^{2}}{2}} \frac{\alpha^{n}}{\sqrt{n !}}, \quad \alpha=|\alpha| e^{i \phi} .
$$

The mean photon number is given by $\bar{n}=\left\langle a^{\dagger} a\right\rangle=|\alpha|^{2}$ and in the following it is assumed to be large $(\bar{n} \gg 1)$. In order to find the time-dependent state vector we choose to solve the eigenvalue problem for $W$ using the photon number states $|n\rangle$, the atomic basis $|\mathrm{gg}\rangle,|\mathrm{ee}\rangle$, and the Bell states

$$
\left|\Psi^{ \pm}\right\rangle=\frac{1}{\sqrt{2}}(|\mathrm{ge}\rangle \pm|\mathrm{eg}\rangle) .
$$

In this basis an arbitrary initial state of the atoms takes the form

$$
\left.|\psi\rangle=c_{\mathrm{g}}|\mathrm{gg}\rangle+c_{-}\left|\Psi^{-}\right\rangle+c_{+}\left|\Psi^{+}\right\rangle+c_{\mathrm{e}} \mid \text { ee }\right\rangle,
$$

where the probability amplitudes fulfill the normalization condition and with the convention $|\mathrm{ge}\rangle=|\mathrm{g}\rangle_{A}|\mathrm{e}\rangle_{B}$. It can be verified by inspection that the set of states $\left\{\left|\Psi^{-}\right\rangle|n\rangle\right\}_{n=0}^{\infty}$ are eigenvectors of $W$ with eigenvalue 0 . The rest of the eigensystem can be evaluated by diagonalizing $3 \times 3$ matrices which in the tripartite basis $\left\{|g g\rangle|n\rangle,\left|\Psi^{+}\right\rangle|n-2\rangle\right.$, |ee $\left.\rangle|n-4\rangle\right\}$, can be written as

$$
W_{n}=g \sqrt{2}\left(\begin{array}{ccc}
0 & \sqrt{n^{2}-n} & 0 \\
\sqrt{n^{2}-n} & 0 & \sqrt{n^{2}-5 n+6} \\
0 & \sqrt{n^{2}-5 n+6} & 0
\end{array}\right) .
$$

Although it is possible to diagonalize these matrices in an exact form, the condition of high mean photon number $|\alpha|^{2} \gg$ 1 will allow us to find compact expressions that are good approximations to the exact results. For instance, the exact nonzero eigenvalues are $w_{n}^{ \pm}= \pm g \sqrt{(2 n-3)^{2}+3}$, but they can be approximated for large values of $n$ by

$$
\tilde{w}_{n}^{ \pm}= \pm g(2 n-3) \text {. }
$$

In this limit, one can find that the orthogonal transformation which diagonalizes each block $W_{n}$ takes the simple form

$$
\tilde{O}_{n}=\frac{1}{2}\left(\begin{array}{ccc}
-\sqrt{2} & 1 & 1 \\
0 & -\sqrt{2} & \sqrt{2} \\
\sqrt{2} & 1 & 1
\end{array}\right)
$$

The evolution operator can also be expressed in terms of matrices of size $3 \times 3$, which can be evaluated using the transformation that diagonalizes the blocks $W_{n}$ of $W$, namely

$$
\tilde{U}_{n}(t)=\tilde{O}_{n}^{\top} \exp \left[-i \operatorname{diag}\left(0,-\tilde{w}_{n}, \tilde{w}_{n}\right) t\right] \tilde{O}_{n}
$$

where $\operatorname{diag}(v)$ represents a diagonal matrix with the elements of $v$ as non-zero entries. With these approximations, the evolution operator has the remarkable simple form

$$
\tilde{U}_{n}(t)=\left(\begin{array}{ccc}
\cos ^{2}\left(\frac{\tilde{w}_{n} t}{2}\right) & \frac{\sin \left(\tilde{w}_{n} t\right)}{i \sqrt{2}} & -\sin ^{2}\left(\frac{\tilde{w}_{n} t}{2}\right) \\
\frac{\sin \left(\tilde{w}_{n} t\right)}{i \sqrt{2}} & \cos \left(\tilde{w}_{n} t\right) & \frac{\sin \left(\tilde{w}_{n} t\right)}{i \sqrt{2}} \\
-\sin ^{2}\left(\frac{\tilde{w}_{n} t}{2}\right) & \frac{\sin \left(\tilde{w}_{n} t\right)}{i \sqrt{2}} & \cos ^{2}\left(\frac{\tilde{w}_{n} t}{2}\right)
\end{array}\right) .
$$

Using these results, one can find that the time evolution of any initial state can be written as

$$
\begin{aligned}
|\Psi(t)\rangle= & a_{0}|\mathrm{gg}, 0\rangle+a_{1}|\mathrm{gg}, 1\rangle+ \\
& \sum_{n=2}^{3}\left(a_{n, t}|\mathrm{gg}, n\rangle+b_{n, t}\left|\Psi^{+}, n-2\right\rangle\right)+ \\
& \left.\sum_{n=4}^{\infty}\left(a_{n, t}|\mathrm{gg}, n\rangle+b_{n, t}\left|\Psi^{+}, n-2\right\rangle+c_{n, t} \mid \text { ee }, n-4\right\rangle\right),
\end{aligned}
$$

where $a_{0}=c_{\mathrm{e}} p_{0}$ and $a_{1}=c_{\mathrm{e}} p_{1}$ are the probability amplitudes of stationary states that are decoupled from the dynamics. The rest of the coefficients can be evaluated with the aid of the evolution operator and are given by

$$
\begin{aligned}
& a_{n, t}=\left(\frac{c_{+}+d_{2 \phi}^{+}}{2} e^{-i \tilde{w}_{n} t}-\frac{c_{+}-d_{2 \phi}^{+}}{2} e^{i \tilde{w}_{n} t}+d_{2 \phi}^{-}\right) \frac{e^{-i 2 \phi} p_{n}}{\sqrt{2}}, \\
& b_{n, t}=\left(\frac{c_{+}+d_{2 \phi}^{+}}{2} e^{-i \tilde{w}_{n} t}+\frac{c_{+}-d_{2 \phi}^{+}}{2} e^{i \tilde{w}_{n} t}\right) p_{n-2}, \\
& c_{n, t}=\left(\frac{c_{+}+d_{2 \phi}^{+}}{2} e^{-i \tilde{w}_{n} t}-\frac{c_{+}-d_{2 \phi}^{+}}{2} e^{i \tilde{w}_{n} t}-d_{2 \phi}^{-}\right) \frac{e^{i 2 \phi} p_{n-4}}{\sqrt{2}} .
\end{aligned}
$$

In the previous expressions we have introduced for notational convenience the coefficients

$$
d_{\phi}^{ \pm}=\frac{c_{\mathrm{g}} e^{i \phi} \pm c_{\mathrm{e}} e^{-i \phi}}{\sqrt{2}}
$$

which are the initial probability amplitudes of the maximally entangled states of the two atoms

$$
\left|\Phi_{\phi}^{ \pm}\right\rangle=\frac{1}{\sqrt{2}}\left(e^{-i \phi}|\mathrm{gg}\rangle \pm e^{i \phi}|\mathrm{ee}\rangle\right)
$$

In order to find a simple expression for the state vector, we use the following approximate relation for the photonic probability amplitudes

$$
p_{n} \approx p_{n-1} e^{i \phi}
$$

With this result one can carry out the summation in Eq. (18) in order the arrive to an approximation of the state vector in terms of coherent states and maximally entangled atomic states, namely

$$
\begin{aligned}
|\Psi(t)\rangle & =\left(c_{-}\left|\Psi^{-}\right\rangle+d_{2 \phi}^{-}\left|\Phi_{2 \phi}^{-}\right\rangle\right)|\alpha\rangle+ \\
& \frac{c_{+}+d_{2 \phi}^{+}}{2} e^{-i g t}\left(\left|\Psi^{+}\right\rangle+\left|\Phi_{2 \phi-4 g t}^{+}\right\rangle\right)\left|e^{-i 2 g t} \alpha\right\rangle+ \\
& \frac{c_{+}-d_{2 \phi}^{+}}{2} e^{i g t}\left(\left|\Psi^{+}\right\rangle-\left|\Phi_{2 \phi+4 g t}^{+}\right\rangle\right)\left|e^{i 2 g t} \alpha\right\rangle .
\end{aligned}
$$



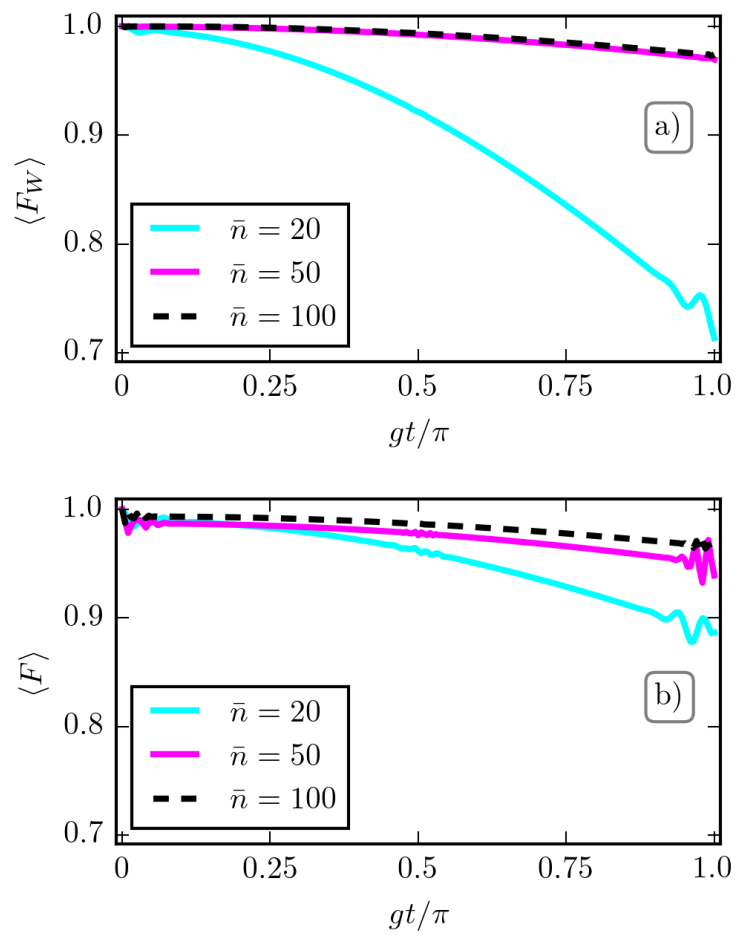

FIG. 2. a) Ensemble average of the fidelity $\left\langle F_{W}\right\rangle$ computed from the exact numerical state vector $\left|\Psi_{W}(t)\right\rangle$ for the effective two-photon Hamiltonian $W$ respect to the numerical solution $\left|\Psi_{\text {exact }}(t)\right\rangle$ corresponding to the full Hamiltonian (1) as a function of the scaled time $g t / \pi$. Three cases are shown: $\bar{n}=20,50,100$. b) Ensemble average of the fidelity $\langle F\rangle$ of the approximate solution $|\Psi(t)\rangle$ (Eq. (23)) also with reference to the state $\left|\Psi_{\text {exact }}(t)\right\rangle$ as function of the scaled time $g t$ with $g_{\mathrm{g}} / \Delta=0.002$. The ensemble average has been performed over $10^{3}$ initial random pure states uniformly distributed according to the Haar measure of $S U(4)$ with $\phi$ a random phase drawn from a uniform distribution $\in[0,2 \pi)$.

A similar expression to this formula was found in [19] for the two-atom Tavis-Cummings model involving more complicated field states that followed the dynamics of a coherent state, but distorting its shape in time. In contrast, the solution in (23) is written in terms of coherent states as a consequence of the linear behavior of the eigenfrequencies of the system for large photon numbers. The full exact solution for the two-photon model was previously reported in Ref. [31] together with a semiclassical approximation in agreement with our findings. There, however, the form in terms of orthogonal Bell states and coherent states was not identified nor its potential application was stressed.

In order to test the validity of our approximation, we have plotted in Fig. 2 a) the fidelity $F_{W}=\left|\left\langle\Psi_{\text {exact }}(t) \mid \Psi_{W}(t)\right\rangle\right|^{2}$ of the exact numerical solution evaluated with the full Hamiltonian in Eq. (1) with respect to the exact numerical state vector computed with the two-photon Hamiltonian in (10) for different values of the average photon number $\bar{n}$. As a comparison we show in Fig. 2 b) the fidelity $F=\left|\left\langle\Psi_{\text {exact }}(t) \mid \Psi(t)\right\rangle\right|^{2}$ with respect to the approximate state vector in terms of coherent states of Eq. (23). In favor of generality, and for both fidelities, we have performed an ensemble average with $10^{3}$ random initial pure states taken from the uniform distribution of $S U(4)$. The phase $\phi$ of the coherent state was randomly obtained from a uniform distribution in the interval $[0,2 \pi)$. It can be noted, that the agreement between dynamics is remarkably good for increasing value of the mean photon number in both situations. Having checked its validity, the solution in Eq. (23) will be the starting point of our subsequent analysis.

\section{COLLAPSE AND REVIVAL OF RABI OSCILLATIONS}

A clear manifestation of the coherent shape of the components of the field state is the perfect revivals of the Rabi oscillations of observables such as the mean value of the operator $S_{\text {ee, }}$, which can be interpreted as the number of atoms in their corresponding excited state. This can be evaluated analytically, for instance for the initial state $\mid$ ee $\rangle|\alpha\rangle$, using our expression (23) as

$$
\left\langle S_{\mathrm{ee}}\right\rangle=1+\operatorname{Re}\left[e^{-|\alpha|^{2}\left(1-e^{i 2 g t}\right)-i 3 g t}\right],
$$

where we have employed the overlap between the relevant coherent states $\left\langle\alpha \mid \alpha e^{ \pm i 2 g t}\right\rangle=e^{-|\alpha|^{2}\left(1-e^{ \pm i 2 g t}\right)}$ which has a Gaussian envelope $\left(1+e^{-2|\alpha|^{2} g^{2} t^{2}}\right) / 2$ for values of time close to zero and in general to $g t=\pi l$, with $l \in \mathbb{N}$. In Fig. 3 we have plotted the numerical exact calculation of $\left\langle S_{\mathrm{ee}}\right\rangle$. As in the case of the standard Jaynes-Cummings interaction, collapses and revivals in this atomic observable are present in the dynamics of the two-photon model (apart from an alternating sign). However, they show a different behavior as they appear in a more compact and regular form, showing almost the complete returning to the initial photonic state in the case of large fields [32]. In the two-photon two-atom model, the time at which revivals appear is independent of $\bar{n}$ and is given by

$$
t_{r} \approx \pi / g
$$

In order to attain $t_{r}$ with the model of Sec. II, the restriction in time of Eq. (9) results in the following condition for the parameters of the model: $g_{\mathrm{e}} \bar{n} \pi \ll \Delta$.

The collapse and revival of Rabi oscillations can also be studied in phase space. This gives a relevant pictorial description of the time-evolution of the field state, whose form will corroborate our approximation in terms of coherent states. We choose to visualize the behavior in terms of the Wigner function [33], a quasi-probability distribution defined as

$$
W(\beta, t)=\frac{1}{\pi^{2}} \int \operatorname{Tr}\left\{\rho_{f}(t) e^{\zeta a^{\dagger}-\zeta^{*} a}\right\} e^{\beta \zeta^{*}-\beta^{*} \zeta} d^{2} \zeta,
$$

with $\beta$ and $\zeta$ being complex numbers and the reduced density operator of the field obtained after tracing out the atomic degrees of freedom, i.e., $\rho_{f}(t)=\operatorname{Tr}_{A, B}|\Psi(t)\rangle\langle\Psi(t)|$. In Fig. 4 we present the Wigner function of the photonic state for three different values of the interaction time, namely $t=0, t=t_{r} / 4$ and $t=t_{r} / 2$. From this representation one can extract relevant dynamical information of the full system. The initial state for 


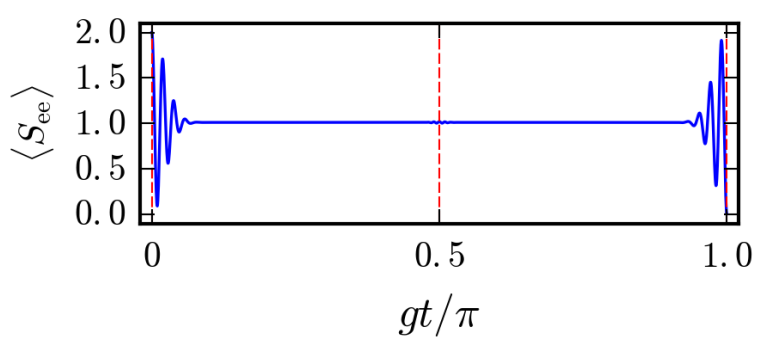

FIG. 3. Collapse and perfect revival of Rabi oscillations in $\left\langle S_{\text {ee }}\right\rangle$ (number of atoms in the excited state) as a function of the scaled time $g t / \pi$. The initial state $\mid$ ee $\rangle|\alpha\rangle$ with $|\alpha|^{2}=50$ evolves under the influence of the effective Hamiltonian (10). An approximate analytical expression for these oscillations is given in Eq. (24).

$t=0$ in Fig. 4 a) corresponds to a coherent state and is represented by a Gaussian distribution in the complex plane. For nonzero values of the interaction time, the field evolves as correlated coherent states, without deforming its circular shape, showing no squeezing during the evolution. The correlated feature is manifested by the interference fringes between the maxima at $t_{r} / 4$ in Fig. 4 b) that disappear at $t_{r} / 2$ in Fig. $4 \mathrm{c}$ ). From Eq. (23), one can evaluate the state vector at half the revival time

$$
\begin{aligned}
\left|\Psi\left(t_{r} / 2\right)\right\rangle & =\left(c_{-}\left|\Psi^{-}\right\rangle+d_{2 \phi}^{-}\left|\Phi_{2 \phi}^{-}\right\rangle\right)|\alpha\rangle \\
& -i\left(c_{+}\left|\Phi_{2 \phi}^{+}\right\rangle+d_{2 \phi}^{+}\left|\Psi^{+}\right\rangle\right)|-\alpha\rangle .
\end{aligned}
$$

Tracing over the atomic degrees of freedom, one finds that the field state corresponds to the mixed state

$$
\begin{aligned}
\rho_{f}= & \left(\left|c_{-}\right|^{2}+\left|d_{2 \phi}^{-}\right|^{2}\right)|\alpha\rangle\langle\alpha|+ \\
& \left(\left|c_{+}\right|^{2}+\left|d_{2 \phi}^{+}\right|^{2}\right)|-\alpha\rangle\langle-\alpha| .
\end{aligned}
$$

This incoherent superposition explains the absence of interference fringes between the two-dimensional Gaussian functions representing opposed coherent states in phase space in Fig. 4 c). The complete state of the system at $t_{r} / 2$, half revival time, given in Eq. (27) will play a key role in what follows. In the next section we will show that multipartite quantum correlations can be generated during the time evolution leading to the formation of tripartite entangled states.

\section{GENERATION OF GHZ STATES}

An immediate application is the possibility to generate maximally entangled three-qubit states using the intrinsic dynamics of the two-photon model. Based on our solution in terms of Bell and coherent states at half revival time in Eq. (27) and setting the coefficients $c_{-}=d_{2 \phi}^{+}=0$, and $c_{+}=\frac{1}{\sqrt{2}}, d_{2 \phi}^{-}=\frac{i}{\sqrt{2}}$, the state vector evaluated at $t_{r} / 2$ takes the following form:

$$
\left|\Psi\left(t_{r} / 2\right)\right\rangle=\frac{i}{\sqrt{2}}\left[\left|\Phi_{2 \phi}^{-}\right\rangle|\alpha\rangle-\left|\Phi_{2 \phi}^{+}\right\rangle|-\alpha\rangle\right] .
$$

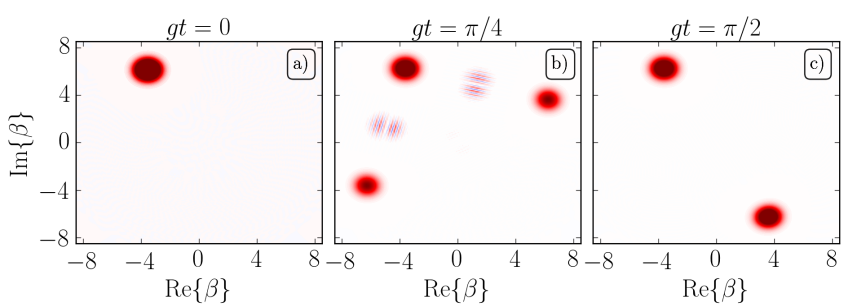

FIG. 4. Wigner function of the optical field for interactions times a) $t_{r}=0$, b) $t_{r}=1 / 4$, c) $t_{r}=1 / 2$. At $t_{r}=1 / 2$ (last snapshot) one can recognize the shape of an incoherent superposition of two coherent states. Parameters are the same as in Fig. 3 with $\phi=2 \pi / 3$.

Looking at the probability amplitudes, the initial state might appear somehow complicated or even entangled, but it is actually an initial tripartite product state with the field in the coherent state $|\alpha\rangle$ and each atom in the state

$$
\left|\varphi_{\phi}\right\rangle=\frac{e^{i \pi / 4}}{\sqrt{2}}\left(e^{-i \phi}|\mathrm{g}\rangle-i e^{i \phi}|\mathrm{e}\rangle\right)
$$

It is therefore a remarkable result that the simple unitary evolution generates a maximally entangled tripartite state with a product state as an input. In order to show that this corresponds to a tripartite entangled state, it is useful to establish an isomorphism between coherent and qubit states for large values of $|\alpha|$. Consider the following even and odd Schrödinger cat states:

$$
|\alpha, \pm\rangle=\frac{1}{\sqrt{2}}(|\alpha\rangle \pm|-\alpha\rangle)
$$

which are eigenstates of the parity operator $\Pi=(-1)^{a^{\dagger} a}$ with eigenvalues \pm 1 that fulfill the condition $\langle\alpha,+\mid \alpha,-\rangle \approx 0$ for $|\alpha| \gg 1$. Even and odd cat states can then be respectively interpreted as the excited and ground states of a two-level system [34]. In fact, one can easily check that the operators:

$$
\begin{aligned}
& \Sigma_{x}=|\alpha,+\rangle\langle\alpha,-|+| \alpha,-\rangle\langle\alpha,+|, \\
& \Sigma_{y}=i(|\alpha,+\rangle\langle\alpha,-|-| \alpha,-\rangle\langle\alpha,+|), \\
& \Sigma_{z}=|\alpha,+\rangle\langle\alpha,+|-| \alpha,-\rangle\langle\alpha,-|,
\end{aligned}
$$

satisfy the same $S U(2)$ algebra $\left[\Sigma_{i}, \Sigma_{j}\right]=2 i \epsilon_{i j k} \Sigma_{k}$ and $\left\{\Sigma_{i}, \Sigma_{j}\right\}=\delta_{i j}$. If we set the phase $\phi=\pi / 4$, the state in Eq. (29) can be rewritten as

$$
|\mathrm{GHZ}\rangle=\frac{1}{\sqrt{2}}(|\mathrm{gg}\rangle|\alpha,-\rangle+|\mathrm{ee}\rangle|\alpha,+\rangle),
$$

which can be immediately recognized as a Greenberger-HorneZeilinger (GHZ) state. It is well known that GHZ states contain one of the two types of tripartite entanglement, and they have been experimentally realized in a variety of physical systems, such as photons, trapped ions, and superconducting qubits [3537]. These states are characterized by the fact that a measurement performed on the third qubit results in an unentangled qubit pair. However, a very interesting fact is that pairwise entanglement can be obtained by performing an appropriate 


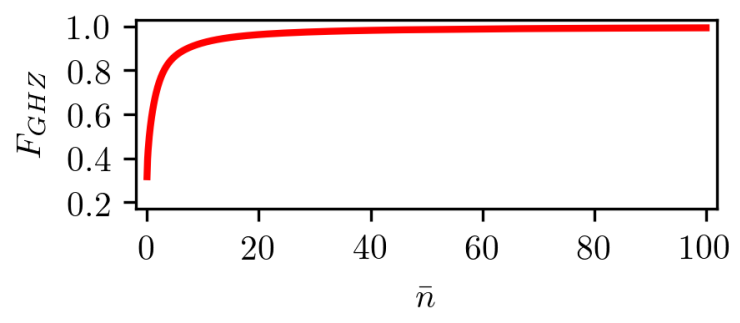

FIG. 5. Fidelity between the generated GHZ state in Eq. (35) and the numerically evaluated state vector, $F_{G H Z}=\left|\left\langle\mathrm{GHZ} \mid \Psi_{\text {exact }}(\pi / 2 g)\right\rangle\right|^{2}$, as a function of the mean photon number.

measurement of the third qubit along some orthogonal direction. From Eq. (35) we can see this by projecting onto the coherent states $| \pm \alpha\rangle$, which automatically leaves the qubit pair in one of the entangled states $\left|\Phi^{ \pm}\right\rangle$. The corresponding fidelity $F_{G H Z}=\left|\left\langle\mathrm{GHZ} \mid \Psi_{\text {exact }}(\pi / 2 g)\right\rangle\right|^{2}$ between the generated GHZ state in Eq. (35) and the exact state vector calculated by numerical means is shown in Fig. 5 for increasing mean photon number. As we have shown, almost unit fidelity GHZ states can be efficiently engineered by the appropriate tuning of the initial conditions.

\section{BELL MEASUREMENT}

In this section we present a scheme to implement a complete Bell measurement based on atomic postselection by letting the atoms interact with two separate cavities and then measuring the field state as depicted in Fig. 6. We elucidate this by first considering the interaction with one cavity for an interaction time equal to half revival time for which the system is left in the state given by Eq. (27). At this time, only two coherent states contribute to the photonic state and are correlated with two orthogonal components of the atomic state. As we are considering the limit of high excitation number, these two coherent states are nearly orthogonal as can be noted from their overlap $\langle\alpha \mid-\alpha\rangle=e^{-2|\alpha|^{2}}$, and therefore can be distinguished with an appropriate measurement scheme [18, 19,38]. For our discussion, we assume that one is able to project the field onto the states $|\alpha\rangle$ or $|-\alpha\rangle$. From the form of the state in Eq. (27), one can note that these projections correspond, respectively, to the following atomic measurement operators

$$
\begin{aligned}
& M_{\phi}^{+}=\left|\Psi^{-}\right\rangle\left\langle\Psi^{-}|+| \Phi_{2 \phi}^{-}\right\rangle\left\langle\Phi_{2 \phi}^{-}\right|, \\
& M_{\phi}^{-}=-i\left|\Phi_{2 \phi}^{+}\right\rangle\left\langle\Psi^{+}|-i| \Psi^{+}\right\rangle\left\langle\Phi_{2 \phi}^{+}\right| .
\end{aligned}
$$

Therefore, projecting onto the photonic state $| \pm \alpha\rangle$ corresponds to implementing the atomic measurement operator $M_{\phi}^{ \pm}$ composed of a rank-two projector and a flip operator, both given in the Bell basis. The appearance of this flip operator is due to the fact that in (27) the initial atomic probability amplitudes of the state in the second row are interchanged. For certain atomic probability amplitudes, the above projection postselect the atoms in an entangled state in a similar fashion as in [39].

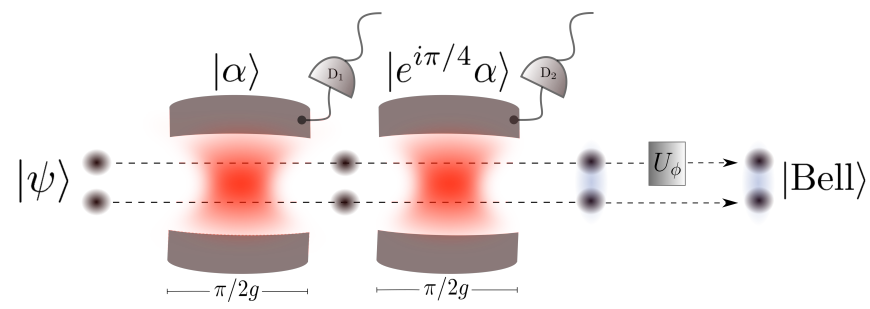

FIG. 6. Schematic visualization of the Bell measurement scheme. Two matter qubits initially described by an arbitrary state $|\psi\rangle$ enter to a sequence of two independent electromagnetic cavities prepared in coherent states. Both qubits couple to each mode for a time $t_{r} / 2$. At the exit of the cavities, a measurement on the field state is performed by detectors $\mathrm{D}_{1}$ and $\mathrm{D}_{2}$. As a final step, a single-qubit unitary $U_{\phi}$ is applied on the first qubit resulting in the postselection of a Bell state.

With the previous result it is not possible to project the atomic states into four orthogonal states, as we have only encountered a rank-two projector and a flip operation in the space of two maximally entangled states. However, one can extend this result with the use of a second cavity, similar to $[18,19]$. For this purpose, one has to let the atoms interact with the field prepared in a coherent state of the form $\left|e^{i \pi / 4} \alpha\right\rangle$, i.e., phase-shifted by $\pi / 4$ from the first coherent state. This can be done, for instance, by letting the atoms interact with a second cavity as depicted in Fig. 6. After an interaction time of $t_{r} / 2$, one would obtain a similar state to the one in Eq. (27), but with rotated coherent states, i.e., $\phi$ replaced by $\phi+\pi / 4$. In this case, projecting onto $\left| \pm e^{i \pi / 4} \alpha\right\rangle$ would correspond to measuring the atoms with a measurement operator $M_{\phi+\pi / 4}^{ \pm}$. Combining this with the previous procedure, one is able to measure the atoms according to the following measurement elements

$$
\begin{aligned}
& M^{++}=M_{\phi+\pi / 4}^{+} M_{\phi}^{+}=\left|\Psi^{-}\right\rangle\left\langle\Psi^{-}\right|, \\
& M^{+-}=M_{\phi+\pi / 4}^{+} M_{\phi}^{-}=-i\left|\Phi_{2 \phi}^{+}\right\rangle\left\langle\Psi^{+}\right|, \\
& M^{-+}=M_{\phi+\pi / 4}^{-} M_{\phi}^{+}=\left|\Psi^{+}\right\rangle\left\langle\Phi_{2 \phi}^{-}\right|, \\
& M^{--}=M_{\phi+\pi / 4}^{-} M_{\phi}^{-}=i\left|\Phi_{2 \phi}^{-}\right\rangle\left\langle\Phi_{2 \phi}^{+}\right| .
\end{aligned}
$$

where we have considered the following relations

$$
\left|\Phi_{\phi+\pi / 2}^{ \pm}\right\rangle=-i\left|\Phi_{\phi}^{\mp}\right\rangle, \quad d_{\phi+\pi / 2}^{ \pm}=i d_{\phi}^{\mp} .
$$

Each measurement element $M^{ \pm \pm}$corresponds to the simultaneous projection onto $| \pm \alpha\rangle$ in the first cavity and onto $\left| \pm e^{i \pi / 4} \alpha\right\rangle$ in the second cavity. It turns out that all $M^{ \pm \pm}$ form the set of measurement operators of a particular positive operator-valued measurement (POVM) [1] that is already good enough to distinguish the four Bell states. However, this does not correspond to a von Neumann measurement, as there are some states that are interchanged during the process. In order to convert this scheme into a von Neumann measurement of the four Bell states, i.e., a Bell measurement, one has to implement a procedure to flip some of the Bell states. Fortunately, this can be accomplished with the help of the following pair of single-atom unitary transformations

$$
\sigma_{\phi}=e^{i \phi}|\mathrm{e}\rangle\left\langle\mathrm{g}\left|+e^{-i \phi}\right| \mathrm{g}\right\rangle\left\langle\mathrm{e}\left|, \quad \sigma_{z}=\right| \mathrm{e}\right\rangle\langle\mathrm{e}|-| \mathrm{g}\rangle\langle\mathrm{g}|,
$$




\begin{tabular}{cccc}
\hline \hline Measured field & Measured field & Postselected & Gate $U_{\phi}$ \\
state in $\mathrm{D}_{1}$ & state in $\mathrm{D}_{2}$ & Bell state & on qubit $A$ \\
$|\alpha\rangle$ & $\left|e^{\mathrm{i} \pi / 4} \alpha\right\rangle$ & $\left|\Psi^{-}\right\rangle$ & $\mathbb{1}$ \\
$|\alpha\rangle$ & $\left|-e^{\mathrm{i} \pi / 4} \alpha\right\rangle$ & $\left|\Psi^{+}\right\rangle$ & $i \sigma_{2 \phi}$ \\
$|-\alpha\rangle$ & $\left|e^{\mathrm{i} \pi / 4} \alpha\right\rangle$ & $\left|\Phi_{2 \phi}^{-}\right\rangle$ & $\sigma_{2 \phi} \sigma_{z}$ \\
$|-\alpha\rangle$ & $\left|-e^{\mathrm{i} \pi / 4} \alpha\right\rangle$ & $\left|\Phi_{2 \phi}^{+}\right\rangle$ & $i \sigma_{z}$ \\
\hline \hline
\end{tabular}

TABLE I. Summary of the quantum protocol indicating the measured field in each cavity, the corresponding post-selected Bell state and the unitary gate that has to be applied to complete a Bell measurement.

that transform the Bell states according to the following rules

$$
\begin{array}{cc}
\sigma_{\phi, A}\left|\Phi_{\phi}^{ \pm}\right\rangle= \pm\left|\Psi^{ \pm}\right\rangle, & \sigma_{z, A}\left|\Psi^{ \pm}\right\rangle=-\left|\Psi^{\mp}\right\rangle \\
\sigma_{\phi, A}\left|\Psi^{ \pm}\right\rangle= \pm\left|\Phi_{\phi}^{ \pm}\right\rangle, & \sigma_{z, A}\left|\Phi_{\phi}^{ \pm}\right\rangle=-\left|\Phi_{\phi}^{\mp}\right\rangle .
\end{array}
$$

Applying these single-qubit gates only to qubit- $A$ in a selective way after the field measurement and according to each outcome, one is able to perform the following Bell-state projections

$$
\begin{aligned}
M^{++} & =\left|\Psi^{-}\right\rangle\left\langle\Psi^{-}\right|, \\
i \sigma_{2 \phi, A} M^{+-} & =\left|\Psi^{+}\right\rangle\left\langle\Psi^{+}\right|, \\
\sigma_{2 \phi, A} \sigma_{z, A} M^{-+} & =\left|\Phi_{2 \phi}^{-}\right\rangle\left\langle\Phi_{2 \phi}^{-}\right|, \\
i \sigma_{z, A} M^{--} & =\left|\Phi_{2 \phi}^{+}\right\rangle\left\langle\Phi_{2 \phi}^{+}\right|,
\end{aligned}
$$

that are required in a complete Bell measurement. The implementation of the selective single-qubit gate is represented in Fig. 6 by the application of the operation $U_{\phi}$ after the interaction with the two cavities. A summary of the protocol with the corresponding single-qubit gate on atom $A$ is presented in table I.

In order to test the protocol, we have carried out numerical simulations to evaluate the fidelity of the postselected atomic states with respect to the corresponding Bell state, i.e., $F_{\text {Bell }}=\left\langle\psi_{\text {Bell }}\left|\rho_{\text {at }}\right| \psi_{\text {Bell }}\right\rangle$, where $\left|\psi_{\text {Bell }}\right\rangle$ stands for one of the four Bell states and $\rho_{\text {at }}$ is the reduced density matrix of the two atoms after implementation of the protocol in Fig. 6. We have also performed an average over $10^{3}$ initial atomic random pure states from a uniform $S U(4)$ distribution in order to produce generic results for two-qubit systems. In Fig. 7 we plot the average fidelity of the numerically obtained Bell states as a function of the mean photon number. We can see that even for relatively small photon numbers, a complete Bell-measurement can be implemented following the proposed protocol. In this case, the only requirement for the mean photon number is to be sufficiently large. This contrasts with previous findings based on the Tavis-Cummings model for which the fidelity of two of the four Bell states is an oscillatory function of the mean photon number, making the protocol functional only for restricted values of the mean photon number [19].

As the protocol is envisioned to work at half of the revival time, it is important to explore the sensitivity of the Bell measurement when the interaction time is closed but not exactly $t_{r} / 2$. In Fig. 8 we plot the average fidelity in a short time window close to half of the revival time for the four postselected

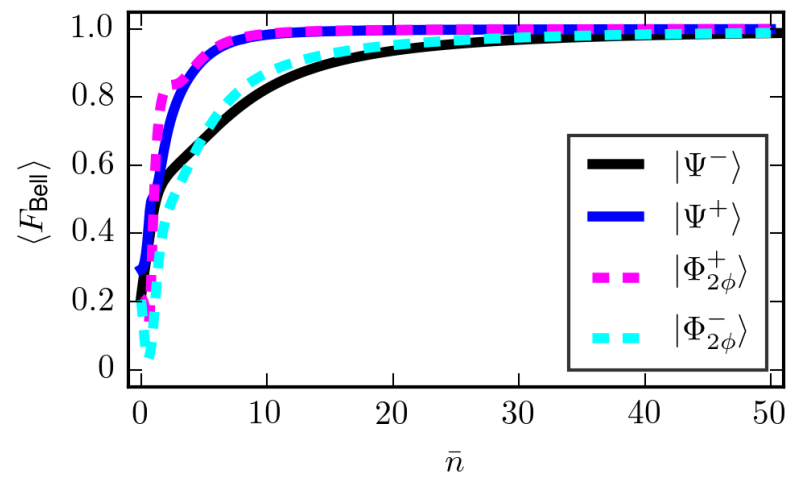

FIG. 7. Ensemble average fidelity $\left\langle F_{\text {Bell }}\right\rangle$ for each postselected Bell state after the application of the protocol as a function of the mean photon number.

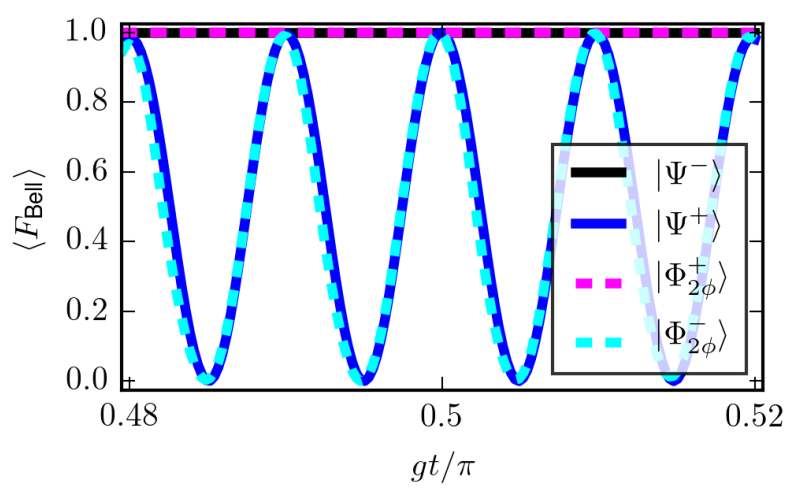

FIG. 8. Ensemble average fidelity $\left\langle F_{\text {Bell }}\right\rangle$ for each postselected Bell state after the application of the protocol around the optimal time $t_{r} / 2$.

Bell states. In this case we have set the mean photon number $\bar{n}=50$, for which we know almost perfect fidelity can be obtained. The results show almost unit fidelity for projecting onto the states $\left|\Psi^{-}\right\rangle$and $\left|\Phi_{2 \phi}^{+}\right\rangle$. The first case can be understood in analogy to the Tavis-Cummings model for which one can show that the state $\left|\Psi^{-}\right\rangle$remains invariant under time evolution [18]. Approximately regular oscillating behavior is found for the other two Bell states near the optimal time $g t=\pi / 2$, with a similar effective frequency roughly given by $g(\bar{n}+1)$. In order to get an idea of a deviation $\epsilon$ allowed in the interaction time $t_{r} / 2 \pm \epsilon$, one can estimate that this possible error must satisfy the condition $|\epsilon| \ll 1 / 2 \pi g(\bar{n}+1)$ for the protocol to work with nearly optimal fidelity.

\section{DISCUSSION}

We now comment on the possibilities of an experimental realization of the above mentioned protocol. In the cavity-QED scenario, experiments using optical conveyor belts to transport neutral laser-cooled cesium atoms into an optical resonator 
have been successfully performed [40, 41]. Adapted to our scheme, the idea would be to transport atoms using a standing wave dipole trap into a sequence of two single mode optical cavities. The effects of losses can be neglected, provided the experiment operates in the strong coupling regime, where the condition $g \gg \gamma, \kappa$ holds, i.e., the qubit-field coupling is much larger than the spontaneous decay rate $\gamma$ of the atoms and the rate accounting for photon losses in the cavities $\kappa$. As in our model the revival time is independent of the mean photon number, at the optimal time these conditions are slightly modified to: $g / \gamma \gg \pi / 2$ and $g / \kappa \gg \pi / 2$. These requirements can be satisfied in current cavity-QED experiments [42].

Similar schemes involving coupled cavities and two-level atoms have been proposed in the context of coherence and entanglement protection in the presence of dissipation [43]. The physical realization of these systems seems to fit very well within the context of the circuit quantum electrodynamics architecture (circiut-QED) [44-46], where transmon qubits can be efficiently coupled to coplanar waveguide cavities. High fidelity preparation of entangled input initial states for the protocol can be in principle engineered using a quantum bus trough a transmission line resonator as described in Ref. [47], and the interaction time of the two-qubit system with the resonator mode can also be switched-off after the corresponding projective measurement of the optical mode, thus implementing all the steps of the algorithm in a single on-chip superconducting circuit.

It is worth commenting on the possible implementation in trapped-ion experiments, as they constitute one of the most successful platforms for quantum simulation and quantum information processing [48]. An interesting simulation based on trapped ions has been recently proposed for emulating the dynamics of the two-photon Rabi model in different coupling regimes [30]. As our protocol is supposed to work in the so called strong-coupling regime, where the qubit frequency is assumed to be very small compared with the qubit-field coupling, its implementation in this particular architecture seems to be feasible with current technology. In this context two identical ions with two internal electronic states are placed in a harmonic trap of a definite frequency. By driving the ions with a laser source at the second red side-band, an effective nonlinear two-phonon coupling can be generated $[29,30]$. After the application of a vibrational RWA one can obtain the trapped-ion Hamiltonian in the interaction picture: $\tilde{H}_{I}=-\Omega \eta^{2}\left(S_{\mathrm{eg}} a^{2}+S_{\mathrm{ge}} a^{\dagger^{2}}\right) / 2$, which can be immediately recognized as our effective Hamiltonian model in Eq. (6) with an effective coupling given by $g_{\text {eff }}=-\Omega \eta^{2} / 2$, being $\Omega$ the Rabi frequency of the laser, and $\eta$ the so-called Lamb-Dicke parameter. The Rabi frequency in trapped-ion experiments lies in the range of $\mathrm{kHz}$ and $\eta \sim 10^{-2}$, which leads to a coupling constant of $g_{\text {eff }} \sim 10^{2} \mathrm{~Hz}$. Taking into account these values, it is possible to estimate the optimal time needed to perform the Bell measurement scheme in a trapped-ion setup from the relation $g_{\text {eff }} t / \pi=1 / 2$, which results in a simulation time of $\sim 10 \mathrm{~ms}$. Typical experiments involving, for instance, optical ${ }^{40} \mathrm{Ca}^{+}$ions have coherence times of $\sim 3 \mathrm{~ms}[49,50]$. These times are still small if a Bell measurement based on the proposed protocol is to be performed. However, continuous dynamical decoupling schemes have been recently proposed in order to achieve long-time coherent dynamics by eliminating magnetic dephasing noise in ion-trap simulators [29], making our proposal for Bell state discrimination more realistic and in reach of current technology.

In our treatment we have assumed, for simplicity, perfect projections onto coherent states in order to discriminate the field components. A more realistic discrimination scheme can be achieved by means of a balanced homodyne detection (BHD), where the field state is superposed with a strong laser beam, known as local oscillator (LO), in a 50/50 beam splitter (BS). By measuring both outputs of the BS via photodetectors, one can achieve the projection onto eigenstates $\left|x, \theta_{L}\right\rangle$ of the field quadratures $x_{\theta_{L}}=\left(a^{\dagger} e^{i \theta_{L}}+a e^{-i \theta_{L}}\right) / 2$, whose eigenvalue $x$ is proportional to the recorded photon difference [51]. The selected quadrature depends on the phase $\theta_{L}$ of the LO. In our protocol, the optimal value is $\theta_{L}=\phi$ in order to distinguish the field components at time $t_{r} / 2$. After the BHD, the field is projected and the atoms collapse to the state $\rho_{\text {at }}=\operatorname{Tr}_{f}[|x, \phi\rangle\langle x, \phi| \rho]$, with the pure state $\rho=\left|\Psi\left(t_{r} / 2\right)\right\rangle\left\langle\Psi\left(t_{r} / 2\right)\right|$ in Eq. (27). With almost unit probability, the postselected atomic state is one of the pure states resulting from the measurement operators $M_{\phi}^{ \pm}$in (36) applied to the state (13). This follows from the fact that the field is solely composed of two coherent states, whose overlaps with the measured field quadrature are given by $\langle x, \phi \mid \pm \alpha\rangle=(2 / \pi)^{1 / 4} \exp \left[-(x \pm|\alpha|)^{2}\right]$ [18], i.e., probable measurements are only registered for values of $x$ close to $\pm|\alpha|$. Therefore, in a BHD of the cavity field, positive (negative) photon counting corresponds to the atomic measurement operator $M_{\phi}^{+}\left(M_{\phi}^{-}\right)$, in Eq. (36), of the initial atomic state given by Eq. (13). Imperfect efficiency $(\varepsilon \in[0,1])$ of the photodetectors can also be considered. In this case the POVM describing the BHD is composed of Gaussian convolution of the ideal projectors onto the field quadratures with variance $\Delta_{\varepsilon}^{2}=(1-\varepsilon) / 4 \varepsilon$ [51]. The recorded probability distribution for each coherent state will then have an effective variance of $\Delta_{\varepsilon}^{2}+1 / 4$. Therefore, one can be confident that BHD is still applicable if $4\left(\Delta_{\varepsilon}^{2}+1 / 4\right)^{1 / 2}$ (four times the standard deviation) is smaller than the distance $|\alpha|$ of each coherent state to the origin. This restriction translates into $\varepsilon>4 /|\alpha|^{2}$ in terms of the photodetection efficiency.

\section{CONCLUSIONS}

We have presented a Bell measurement scheme on atomic qubits that interact with the electromagnetic field contained in two separate cavities via two-photon processes in a two-stage Ramsey-type setup. The protocol is based on the two-atom two-photon Dicke model in the limit of large photon number for initial coherent states of the field. Under such conditions, we have derived an approximate solution in terms of atomic Bell states and photonic coherent states, allowing the identification of an appropriate Bell-measurement protocol via coherent state discrimination in two separate cavities. In contrast with previous proposed protocols $[18,19]$ based on multiphoton states, the one presented here allows a complete discrimination of the 
four atomic Bell states, i.e. a 100\%-efficient Bell measurement that we have numerically confirmed by computing the average fidelity over random initial states. The robustness of the protocol as a function of the interaction time has been tested and the corresponding condition for a possible error in terms of the mean photon number was estimated. By analyzing the time-dependent state of the full system, we have also demonstrated that tripartite entangled GHZ states can be naturally generated by the unitary dynamics of the two-photon model, a possibility that can be further exploited in other quantum information protocols. It is worth stressing that the complete projection onto the full Bell basis is possible as a consequence of the perfect discrimination of two separate components of the evolved field which in turn relies on the perfect revivals of Rabi oscillations in the two photon model.

\section{ACKNOWLEDGMENTS}

We thank Ralf Betzholz and Thomas H. Seligman for enriching discussions. CGG is grateful to CONACYT for financial support under doctoral fellowship 385108 and research grant 219993, and to UNAM/DGAPA/PAPIIT for the research grants IG100616 and IN103017. JMT acknowledges support by PRODEP-SEP project 511-6/18-9344.
[1] M. A. Nielsen and I. Chuang, Quantum computation and quantum information (Cambridge University Press, 2000).

[2] D. Bouwmeester, J.W. Pan, K. Mattle, M. Eibl, H. Weinfurter and A. Zeilinger, Nature 390, 575 (1997).

[3] P. van Loock, T. D. Ladd, K. Sanaka, F. Yamaguchi, Kae Nemoto, W. J. Munro and Y. Yamamoto, Phys. Rev. Lett. 96, 240501 (2006).

[4] S. Pirandola, J. Eisert, C. Weedbrook, A. Furusawa and S. L. Braunstein, Nat. Photonics 9, 641 (2015).

[5] M. Michler, K. Mattle, H. Weinfurter and A. Zeilinger, Phys. Rev. A 53, R1209 (1996).

[6] N. Lütkenhaus, J. Calsamiglia and K.-A. Suominen, Phys. Rev. A 59, 3295 (1999).

[7] J. Calsamiglia and N. Lütkenhaus, App. Phys. B 72, 67 (2001).

[8] F. Ewert and P. van Loock, Phys. Rev. Lett. 113, 140403 (2014).

[9] W. P. Grice, Phys. Rev. A 84, 042331 (2011).

[10] C. H. Bennett, Gilles Brassard, Claude Crépeau, Richard Jozsa, Asher Peres and William K. Wootters, Phys. Rev. Lett. 70, 1895 (1993).

[11] Y.-H. Kim, S. P. Kulik and Y. Shih, Phys. Rev. Lett. 86, 1370 (2001).

[12] M. Riebe, H. Häffner, C. F. Roos, W. Hänsel, J. Benhelm, G. P. T. Lancaster, T. W. Körber, C. Becher, F. Schmidt-Kaler, D. F. V. James and R. Blatt, Nature 429, 734 (2004).

[13] M. D. Barrett, J. Chiaverini, T. Schaetz, J. Britton, W. M. Itano, J. D. Jost, E. Knill, C. Langer, D. Leibfried, R. Ozeri and D. J. Wineland, Nature 429, 737 (2004).

[14] M. Riebe, M. Chwalla, J. Benhelm, H. Häffner, W. Hänsel, C. F. Roos and R. Blatt, New J. Phys. 9, 211 (2007).

[15] F. Schmidt-Kaler, H. Häffner, S. Gulde, M. Riebe, G.P.T. Lancaster, T. Deuschle, C. Becher, W. Hänsel, J. Eschner, C.F. Roos and R. Blatt, Appl. Phys. B 77, 789 (2003).

[16] F. Schmidt-Kaler, H. Häffner, M. Riebe, S. Gulde, G. P. T. Lancaster, T. Deuschle, C. Becher, C. F. Roos, J. Eschner and Rainer Blatt, Nature 422, 408 (2003).

[17] C. Nölleke, A. Neuzner, A. Reiserer, C. Hahn, G. Rempe and S. Ritter, Phys. Rev. Lett. 110, 140403 (2013).

[18] J. M. Torres, J. Z. Bernád and G. Alber, Phys. Rev. A 90, 012304 (2014).

[19] J. M. Torres, J. Z. Bernád and G. Alber, App. Phys. B 122, 117 (2016).

[20] J. Z. Bernád, Phys. Rev. A 96, 052329 (2017).

[21] C.E.A. Jarvis, D.A. Rodrigues, B.L. Györffy, T.P. Spiller, A.J. Short and J.F. Annett, New J. Phys. 11, 103047 (2009).
[22] J. H. Eberly, N. B. Narozhny and J. J. Sanchez-Mondragon Phys. Rev. Lett. 44, 1323 (1980).

[23] A. B. Klimov, J. Negro, R. Farias and S. M. Chumakov, J. Opt. B 1, 562 (1999).

[24] P. Alsing and M. S. Zubairy, J. Opt. Soc. Am. B 4, 177 (1987).

[25] C. V. Sukumar and B. Buck, Phys. Lett. A 83, 211 (1981).

[26] A. H. Toor and M. S. Zubairy, Phys. Rev. A 45, 4951 (1992).

[27] I. Jex, Quantum Opt., 2, 443 (1990).

[28] M. Brune, J. M. Raimond and S. Haroche, Phys. Rev. A 35, 154 (1987).

[29] R. Puebla, M.J. Hwang, J. Casanova and M. B. Plenio, Phys. Rev. A 95, 063844 (2017).

[30] S. Felicetti, D. Z. Rossatto, E. Rico, E. Solano and P. Forn-Díaz, Phys. Rev. A 97, 013851 (2018).

[31] E. K. Bashkirov Phys. Scr. 82, 015401 (2010).

[32] R. R. Puri and R. K. Bullough, J. Opt. Soc. Am. B 5, 2021 (1988).

[33] K. Vogel and H. Risken Phys. Rev. A 40, 2847(R) (1989).

[34] C. Gerry, Phys. Rev. A 54, R2529 (1996).

[35] D. Bouwmeester, J.W. Pan, M. Daniell, H. Weinfurter and A. Zeilinger, Phys. Rev. Lett. 82, 1345 (1999).

[36] T. Monz, P. Schindler, J. T. Barreiro, M. Chwalla, D. Nigg, W. A. Coish, M. Harlander, W. Hänsel, M. Hennrich and R. Blatt, Phys. Rev. Lett. 106, 130506 (2011).

[37] J. Kelly, R. Barends, A. G. Fowler, A. Megrant et al., Nature 519, 66 (2015).

[38] C. Wittmann, M. Takeoka, K.N. Cassemiro, M. Sasaki, G. Leuchs and U.L. Andersen, Phys. Rev. Lett. 101, 210501 (2008).

[39] J. Z. Bernád, J. M. Torres, L. Kunz and G. Alber, Phys. Rev. A 93, 032317 (2016).

[40] R. Reimann, W. Alt, T. Macha, D. Meschede, N. Thau, S. Yoon and L. Ratschbacher, New J. Phys. 16, 113042 (2014).

[41] R. Reimann, W. Alt, T. Kampschulte, T. Macha, L. Ratschbacher, N. Thau, S. Yoon and D. Meschede, Phys. Rev. Lett. 114, 023601 (2015).

[42] C. Hamsen, K. N. Tolazzi, T. Wilk and G. Rempe Phys. Rev. Lett. 118, 133604 (2017).

[43] Z.-X. Man, Y.-J. Xia and R. Lo Franco, Sci. Rep. 5, 13843 (2015).

[44] A. Blais, R.-S. Huang, A. Wallraff, S. M. Girvin and R. J. Schoelkopf, Phys. Rev. A 69, 062320 (2004).

[45] N. T. Bronn, E. Magesan, N. A. Masluk, J. M. Chow, J. M. Gambetta and M. Steffen, IEEE Trans. Appl. Supercond. 25, 1700410 (2015). 
[46] X. Gu, A. F. Kockum, A. Miranowicz, Y-x. Liu and F. Nori, Phys. Rep. 718, 1 (2017).

[47] L. DiCarlo, J. M. Chow, J. M. Gambetta, Lev S. Bishop, B. R. Johnson, D. I. Schuster, J. Majer, A. Blais, L. Frunzio, S. M. Girvin and R. J. Schoelkopf, Nature 460, 240 (2009).

[48] D. Leibfried, R. Blatt, C. Monroe and D. Wineland, Rev. Mod. Phys. 75, 281 (2003).

[49] R. Gerritsma, B. P. Lanyon, G. Kirchmair, F. Zähringer, C. Hempel, J. Casanova, J. J. García-Ripoll, E. Solano, R. Blatt and C. F. Roos, Phys. Rev. Lett. 106, 060503 (2011).

[50] N. Timoney, I. Baumgart, M. Johanning, A. F. Varn, M. B. Plenio, A. Retzker and Ch. Wunderlich, Nature 476, 185 (2011).

[51] G. M. D’Ariano, M. G. A. Paris, and M. F. Sacchi, Adv. Imaging Electron Phys. 128, 205 (2003). 